\section{Development of a scoring system for the diagnosis of tuberculous lymphadenitis}

B.M.M. Ngwira, S.Chaguluka, D.K.Warndorff, K.Branson, S.B. Lucas, P.E.M. Fine

\section{Introduction}

The HIV/ AIDS epidemic has been associated with an increase in smear-negative pulmonary and extrapulmonary registered tuberculosis (TB) cases in many countries including Malawi[1]. Tuberculous lymphadenitis accounts for approximately $25 \%$ of the extrapulmonary cases [2]. Up to $50 \%$ of HIV infected individuals will develop persistent generalised lymphadenopathy (PGL) in the course of their disease [3]. It can be a challenge to distinguish between PGL and lymphadenopathy due to TB especially in resource poor settings without histopathological and cytological diagnostic facilities. The aim of this prospective study was to develop and evaluate a scoring system based on history and physical examination for the diagnosis of glandular TB that would be applicable in settings without laboratory backup in Malawi.

\section{Methods}

Study site

This study was conducted in Karonga District- northern Malawi in the context of the Karonga Prevention Study (KPS), a large epidemiological study of mycobacterial diseases and HIV. Since 1986 the KPS has been responsible for "case-finding" and "case-holding" for both TB and leprosy as part of followup for a large vaccine trial[4]. HIV prevalence among antenatal women and smear-positive pulmonary TB cases were approximately $10 \%$ and $60 \%$, respectively, in the late 1990s [5].

\section{Recruitment}

From October 1997 to October 1999 individuals found with significant lymphadenopathy $(>2 \mathrm{~cm})$ in at least one extrainguinal site were invited to join the study. These were identified during routine standard full body examinations (for leprosy) amongst outpatients, inpatients (both at the District Hospital and Health Centres across the district) and in households during field based studies. Each consenting individual had a "glandular TB suspect" form (figure 1) completed. The various signs carried numerical weights that were assigned on the basis of literature review and a pilot investigation. Once the form was filled in, the paramedic performing the examination calculated a total score and this determined subsequent action. If the total score was less than 10, the individual was asked to return for review after 3 months. If it was between 10 and 19 a week's course of an antibiotic (cotrimoxazole or doxycycline) treatment trial was given, with instructions to return after completing the dosage. Individuals who scored 20 or greater were immediately referred to Karonga District Hospital (KDH) for further investigations.

\section{Assessment of patients}

At $\mathrm{KDH}$, individual verbal consent for biopsy was obtained. For those less than 16 years old a parent or guardian consented. Pre-test counselling for HIV was given to all patients before a blood sample was drawn. Post-test counselling was given to those who wanted to know their status before disclosing the result. Each individual had a fine needle aspiration (FNA) 14
Figure 1

GLANDULAR TB SUSPECT FORM

Nane:
Sex $\mathbf{~ F ~}$
Reason of presentation presentation

\begin{tabular}{|c|c|c|c|c|}
\hline \multicolumn{5}{|l|}{ HISTORY } \\
\hline Fever & None $(0)$ & \multicolumn{2}{|c|}{$1-14$ days (1) } & $>14$ days $(2)$ \\
\hline Night sweats & None $(0)$ & \multicolumn{2}{|c|}{ 1-14 days (1) } & $>14$ days $(2)$ \\
\hline Weight loss & None $(3)$ & \multicolumn{2}{|c|}{ moderate (2) } & Severe (1) \\
\hline Pain in node & None (3) & \multicolumn{2}{|c|}{ Moderate (2) } & Severe (1) \\
\hline Growth ( 2 weeks) & None (2) & \multicolumn{2}{|c|}{ Moderate (1) } & Much (0) \\
\hline TB contact & None known $(0)$ & \multicolumn{2}{|c|}{$H / h, F a m>2 y r s$ (2) } & $\begin{array}{l}\text { H/h Fam } \\
<2 \text { yrs }(3)\end{array}$ \\
\hline \multicolumn{5}{|l|}{ CLINICAL EXAM } \\
\hline General conditions & Fair & \multicolumn{2}{|l|}{ Sick } & Very ill \\
\hline \multirow[t]{6}{*}{ Enlarged glands } & Cervical (C) & \multicolumn{2}{|l|}{$\mathrm{L} \mathrm{Y} / \mathrm{N}$} & $\mathrm{R} Y / \mathrm{N}$ \\
\hline & Submandibular (\$) & \multicolumn{2}{|l|}{$\mathrm{L}, \mathrm{Y} / \mathrm{N}$} & R Y/N \\
\hline & Submental (M) & \multicolumn{2}{|l|}{$\mathrm{Y} / \mathrm{N}$} & \\
\hline & Axillary (A) & \multicolumn{2}{|l|}{$\mathrm{L} \mathrm{Y} / \mathrm{N}$} & $\mathrm{R} Y / N$ \\
\hline & Epitrochlear (E) & \multicolumn{2}{|l|}{ L. $Y / N$} & R Y/N \\
\hline & Inguinal (I) & \multicolumn{2}{|l|}{$L \quad Y / N$} & $\mathrm{R}$ Y/N \\
\hline \multirow[t]{2}{*}{ Symmetry: } & Site: No (3) & Yes & \multicolumn{2}{|c|}{ (then consider size) } \\
\hline & Size: & \multicolumn{2}{|l|}{ Equal (1) } & Unequal) (2) \\
\hline \multicolumn{5}{|c|}{ Maximum score for any node group for each characteristic: } \\
\hline Consistency & Soft $(0)$ & \multirow[t]{3}{*}{ Fim $(1)$} & \multirow[t]{3}{*}{ Hard (2) } & \multirow{3}{*}{$\begin{array}{l}\text { Fluctuant (3) } \\
\text { Fixed (3) }\end{array}$} \\
\hline Mobility & Mobile (1) Doubtful (2 & & & \\
\hline Fused/Matted No (1) & Doubtful (2) Yes (3) & & & \\
\hline \multicolumn{5}{|c|}{ Note: Group = one or more nodes at a particular site } \\
\hline TOTAL SCORE & ACTION: & $\begin{aligned} \text { IF }< & 10 \\
& 10-20 \\
& >20\end{aligned}$ & \multicolumn{2}{|c|}{$\begin{array}{l}\text { Treatment trial } \\
\text { Refer to hospital }\end{array}$} \\
\hline
\end{tabular}

TREATMENT TRIAL

Antibiotic

Dates 1 . to 1

Result: If no change, or doubtful, MO to review; else discharge.

Date $1,1+$ Staff code $+\ldots$

v2 $17 / 11 / 97$

performed using a 23 gauge needle and $20 \mathrm{ml}$ syringe mounted onto a suction gun. The material obtained was applied onto a slide, then thinly spread using a second slide, making two films in the process. Both slides were labeled, air dried and then stored in a slide box. The material remaining in the syringe was applied directly onto solid Lowenstein Jensen (L-I) media. An excision biopsy was then performed under sterile conditions and local anaesthetic. The node removed was divided into two portions, and the cut surface examined macroscopically for caseation. One section was stored in a bottle containing formalin for histology. The other half was put in a plain sterile container and kept frozen. If caseation was seen at FNA and/or excision biopsy the patient was started on antituberculosis treatment according to the Malawi National TB programme treatment guidelines [6]. A blood sample for HIV testing was collected at the same time. All specimens were transported to the KPS laboratory, at the project's headquarters in Chilumba, for further processing.

\section{Laboratory procedures}

At the laboratory one of the FNA slides was stained with auramine-phenol and examined using fluorescence microscopy. Scantly positive slides were destained and restained by ZiehlNielsen for confirmation. The frozen section was thawed, a smear made and the rest was crushed, decontaminated and 2 cultures set up. The cultures included pyruvate-rich media to encourage growth of Mycobacterium bovis [7]. These cultures (including the one made from FNA material) were examined fortnightly for 8 weeks. Those with growth and colony morphology suggestive of $M$ tuberculosis were sent to the 


\section{ARTICLE}

PHLS Mycobacterium Reference Laboratory in Dulwich, London, UK for species identification and drug sensitivity testing. The section fixed in formalin and the remaining slide from FNA were sent to St Thomas' Hospital, London for histopathological and cytological examination respectively (by SBL).

HIV testing was carried out following a standard KPS protocol involving dual testing by both particle agglutination (Edgware- Mast Diagnostics-modified Serodia at two dilutions) and by sandwich ELISA (Organon- Vironostika Uniform II plus 0 test-Organon Teknik ). Only concordant results are reported to the patient. In case of discordant results both tests are repeated in duplicate on serum reobtained from the original specimen.

\section{Data management}

All data were double entered and verified at the Project Headquarters in Karonga using Epi info (version 6; Center for Disease Control (CDC); U.S.A) and Foxpro (version 2.6A) software. Analyses were performed in Stata (version 6, Stata Corporation, Texas, USA).

\section{Diagnostic criteria}

For this analysis, a case was considered "confirmed" if histological findings were consistent (pathognomonic) with TB beyond reasonable doubt and/or $M$. tuberculosis was identified from culture.

\section{Results}

Overall 166 patients were enrolled into the trial as shown in figure 2. Of these 52 did not have further investigations ( 28 improved on treatment trial, 3 were too ill for biopsy and 21 did not return for review). Of the remaining 114 patients, 105 had excision biopsy and FNA performed (75 TB confirmed), 6 had excision biopsy only ( 2 TB confirmed) and 3 had FNA only ( 2 TB confirmed bacteriologically). The mean age of the confirmed TB cases was 28.4 years (SD 10.8, range $0-52$ years). The age and sex distribution of individuals with a confirmed TB lymphadenitis diagnosis (histologically and / or bacteriologically) is shown in figure 3 .

Microscopy results of FNA and frozen section slides are available on 72 confirmed TB cases. Of these 11(15.3\%) were positive on a smear made from FNA material (of these 5 were negative on frozen section smears) and 15 (20.8\%) were positive from frozen section smears (of these 7 were negative on the FNA smear). Of the 75 cultures set up from confirmed
Figure 3

\section{Age and sex distribution of confirmed TB \\ lymphadenitis cases}

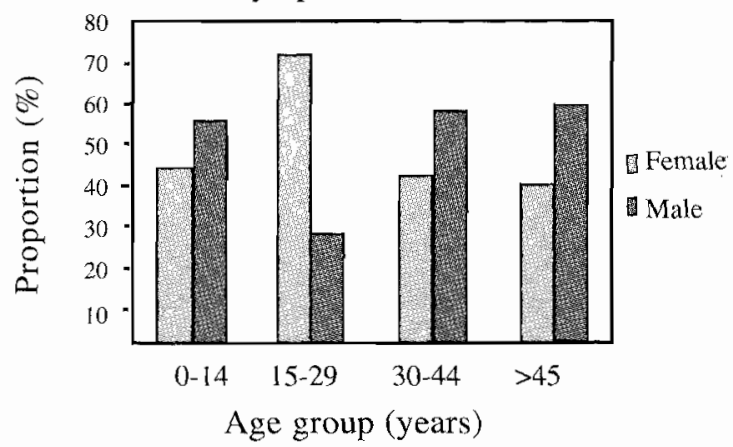

TB cases, 32 (42\%) yielded $M$. tuberculosis from the FNA culture (11 of these had no growth from the frozen section) whereas $36(50 \%)$ had MTB identified from the frozen section cultures ( 9 of these had no growth on the FNA culture). Of note $15(20 \%)$ of the FNA cultures were contaminated compared to $3(4 \%)$ from the frozen section.

Macroscopic examination records were available for 74 confirmed TB cases. Out of these, 64 (85.6\%) were correctly identified as having TB based on caseation. Of ten that were not recognised on this basis, one was recorded as having no caseation and 9 were classified as "doubtful". Of the 34 non-TB cases, $2(5.9 \%)$ were identified as having caseation on naked eye (macroscopic) examination (1 Kaposi Sarcoma and 1 NonHodgkin's Lymphoma). Of the 63 TB confirmed cases with an HIV result, $54(85.7 \%)$ were seropositive. All eight cases of Kaposi sarcoma were HIV seropositive.

The distribution of total points scored by individuals with a confirmed diagnosis is shown in figure 4 . The distribution of clinical features amongst individuals with a confirmed diagnosis is shown in the table. Significant $p$ values are highlighted.

\section{Discussion}

This is a preliminary analysis. A detailed multivariate analysis of diagnostic scores and results is underway.

Figure 2: Trial Profile

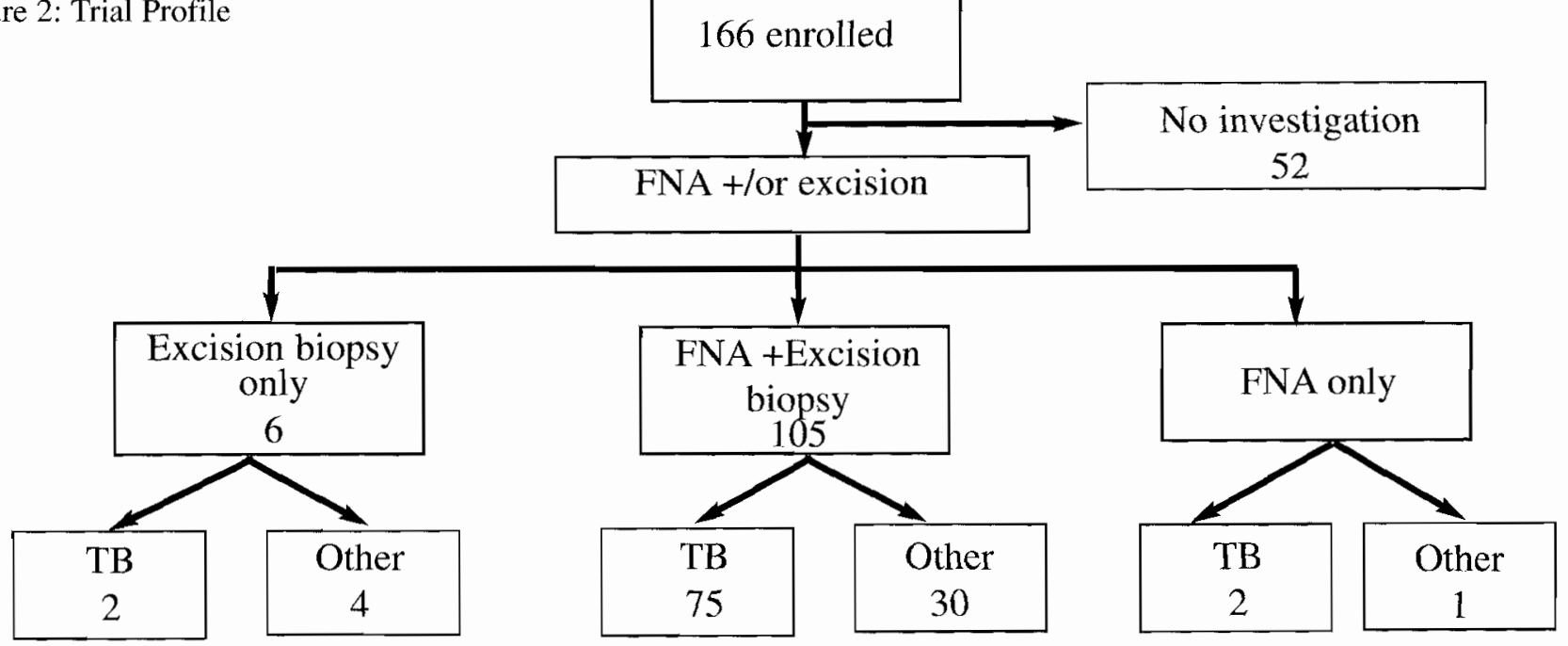


Flgure 4:

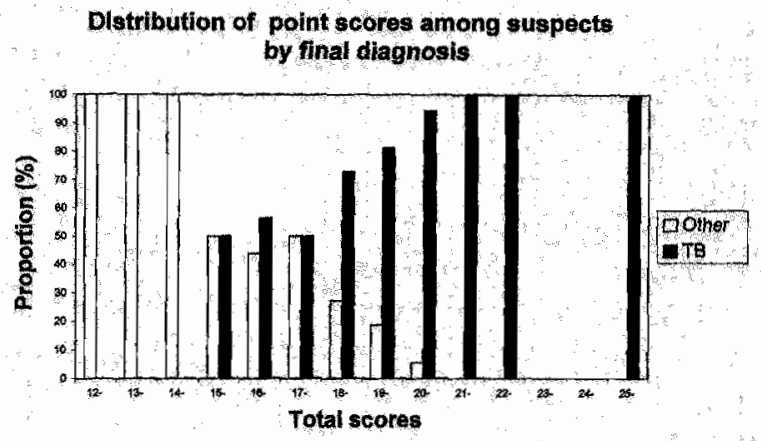

Overall there was no difference in the proportion of confirmed TB cases by sex $(p=0.16)$ or by age $(p=0.13)$. However we note that there is marked female excess in young adults (15-29 years) with glandular TB. This distribution mirrors that of HIV in Malawi[8]. The high HIV seroprevalence amongst glandular TB patients has implications for care. The relatively low proportion of smears positive from FNA and biopsy sections are similar to those reported previously [9] [10]. One possible explanation is that we included individuals from outpatient clinics and from the community, hence in early stages of their disease with low bacillary load. It is worth adding that nodes which yielded much material at FNA were more likely to be smear positive $(\mathrm{p}=0.02)$ and culture positive $(\mathrm{p}=0.04)$ than were the nodes that yielded little material. The high contamination rate $(20 \%)$ of the FNA cultures is not unexpected as these cultures were set up in the operating room without any decontamination process. Notably there was no significant difference in the total number of positive cultures between FNA and frozen section cultures $(p=0.8)$. This study confirms the rarity of human $M$ bovis disease in Karonga, as all the positive cultures were identified as M. tuberculosis. This is despite setting up cultures on pyruvate-rich media to encourage growth of $M$ bovis.

Sensitivity and specificity of macroscopic examination of the sectioned nodes were $97.0 \%$ and $76.3 \%$ respectively. The low specificity achieved here is of concern. This is due to the 9 cases that were classified as "doubtful" for caseation. Of these, 7 had an HIV result and 6 were seropositive. These atypical macroscopic and histological features of tuberculous lymphadenitis in HIV infected individuals have been described elsewhere[11]. The potential for error in macroscopic examination for caseation in malignancy (e.g. Kaposi Sarcoma, Hodgkin's) is known [12]. This calls for particular attention in examining for typical Kaposi sarcoma mucocutaneous lesions in individuals suspected of tuberculous lymphadenitis.
With regard to the performance of the scoring system, confirmed tuberculous lymphadenitis patients tended to achieve higher total scores than those patients with a non-TB diagnosis ( $(2$ test for heterogeneity $\mathrm{p}=<0.01)$. Among the clinical features fever, night sweats, weight loss, growth, anatomical distribution and consistency achieved statistical significant level $(p<0.05)$. Logistic regression analysis will determine the best combination of these features for optimum performance. A possible limitation of this study could be that since experienced paramedics carried out enrolment, they recruited clients in whom a TB diagnosis was most likely. Thus this study could be measuring their clinical acumen. It is imperative therefore that this tool be evaluated in other settings of equally high HIV prevalence.

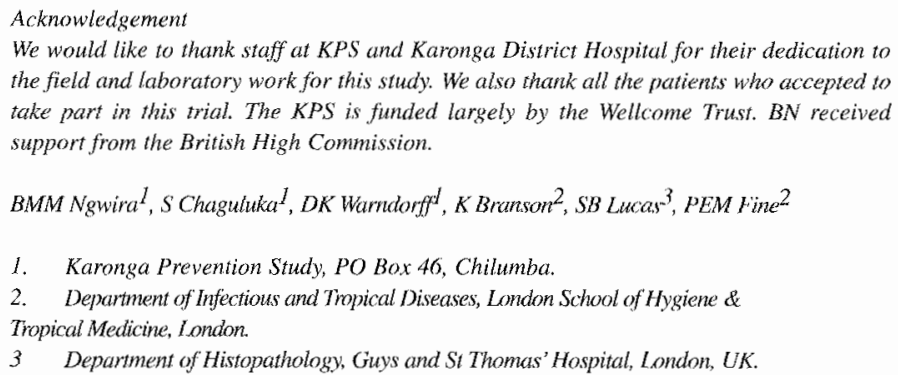

We would like to thank staff at KPS and Karong a District Hospital for their dedication to the field and laboratory work for this study. We also thank all the patients who accepted to take part in this trial. The KPS is funded largely by the Wellcome Trust. BN received support from the British High Commission.

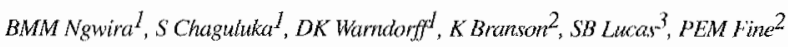

3 Department of Histopathology, Guys and St Thomas' Hospital, London, UK.

Correspondence to: Dr B.M.Ngwira, Malawi Project,

Department of Infectious and Tropical Diseases,

London School of Hygiene \& Tropical Medicine, Keppel Street,

London WCIE 7HT, UK. Phone + 2079272631 . Fax +2076374314

E-mail: Bagrey.Ngwira@lshtm.ac.uk

\section{Reference}

1. Hargraves NJ, Kadzakumanja $O$, Phiri $S$ et al. What causes smear-negative pulmonary tuberculusis in Malawi, an area of high HIV scroprevalence. Int $J$ TubercLung Dis 2001; 5: 113-122.

2. Kwanjana JH, Harries $\mathrm{AD}$, Hargreaves $\mathrm{NJ}$ et al. Sputum-smear examination in patients with extrapulmonary tuberculosis in Malawi. Trans R Soc Trop Med Hyg 2000; 94 : 395-8.

3. Harries AD, Maher D. TB/HIV clinical manual. World Health Organisation 1996.

4. The Karonga Prevention Trial Group. Randomisced controlled trial of single BCG, sepeated BCG and killed Mycobacterium leprac for prevention of leprosy and ubberculosis in Malawi. Lancet 1996; 348: 1724.

5. Glynn JR, Ponnighaus J, Crampin A et al. The development of the HIV epidemic in Karonga District, Malawi. AIDS 2001; 15: 2025-29.

6. Malawi Ministry of Health and Population. Manual of the National Tuberculosis Programme. 1996. 7. Collins $\mathrm{CH}$, Grange IM, Yates MD. Tuberculosis bacteriology: organisation and practice. ButterwothHeinemann 1997

8. UNAIDS. Epidemiological fact sheets on HN/AIDS and sexually transmitted infections: Malawi. WWW. UNAIDS.ORG, 2000.

9. Bckedam HJ, Boeree M, Kamenya Aet al, Tuberculous lymphadeniti, a diagnostic problem in areas of high prevalence of HIV and tuberculosis. Trans R Soc Trop Med Hyg 1997; 91; 294.97.

10. Vetbery A, Lucas S. Tuberculosis or persistent gencralised lymphadenopathy in HIV disease? Lancet 1991; 337: 56-57.

11. Nambuya A, Sewankambo N, Mugerwa J. Tuberculous lymphadenitis associated with HIV in Uganda. J Clin Path 1988; 44: 93-96.

12. Bem C. The value of naked eye examination of biopsed lymph nodes in the diagnosis of tuberculous lymphadenitis. Tropical Doctor 1996;26: 10-13.

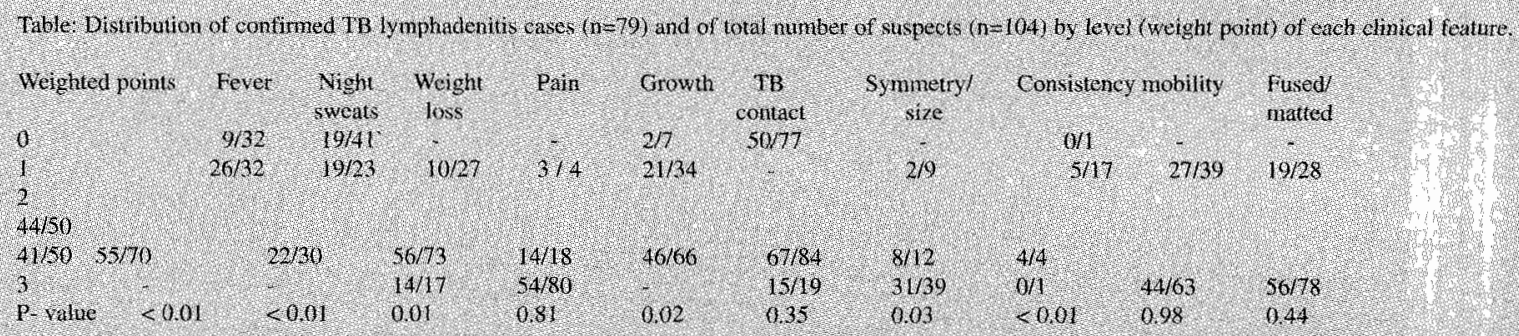

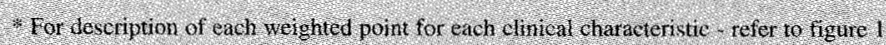

